\title{
From Design to in vivo Active Organometallic- containing Antimycotic Agents
}

Riccardo Rubbiani, ${ }^{a *}$ Tobias Weil, ${ }^{b *}$ Noemi Tocci, ${ }^{b}$ Luciano Mastrobuoni, ${ }^{a}$ Severin Jeger, ${ }^{a}$ Marco Moretto, ${ }^{\mathrm{c}}$ James Ng, ${ }^{\mathrm{d}}$ Yan Lin, ${ }^{\mathrm{d}}$ Jeannine Hess, ${ }^{\text {a Stefano Ferrari, }}{ }^{\mathrm{e}}$ Andres Kaech, ${ }^{\mathrm{f}}$ Luke Young, ${ }^{\mathrm{g}}$ John Spencer, ${ }^{g}$ Anthony L. Moore, ${ }^{h}$ Kevin Cariou, ${ }^{d *}$ Monica Borghi, ${ }^{h}$ Renga Giorgia, 'Luigina Romani, ${ }^{i}$ and Gilles Gasser ${ }^{\mathrm{d}, *}$

a) Department of Chemistry, University of Zurich, Winterthurerstrasse 190, 8057 Zurich, Switzerland

b) Department of Food Quality and Nutrition, Research and Innovation Centre, Fondazione Edmund Mach Via E. Mach 1, 38010 San Michele all'Adige, Italy

c) Unit of Computational Biology, Research and Innovation Centre, Fondazione Edmund Mach

Via E. Mach 1, 38010 San Michele all'Adige, Italy

d) Chimie ParisTech, PSL University, CNRS, Institute of Chemistry for Life and Health Sciences, Laboratory for Inorganic Chemical Biology, 75005 Paris, France.

e) Institute of Molecular Cancer Research, University of Zurich, Winterthurerstrasse 190, 8057 Zurich, Switzerland

f) Center for Microscopy and Image Analysis, University of Zurich, Winterthurerstrasse 190, 8057 Zurich, Switzerland

g) Department of Chemistry, School of Life Sciences, University of Sussex, Brighton BN1 9QJ, UK.

h) Biochemistry \& Biomedicine, School of Life Sciences, University of Sussex, Brighton BN1 9QG, UK.

i) University of Perugia, Department of Experimental Medicine, Piazzale Lucio Severi - Polo Unico Sant'Andrea delle Fratte, 06132 Perugia, Italy. 


\begin{abstract}
Fungal infections are an alarming global problem, most importantly for immunocompromised patients in a hospital environment. The appearance of multidrug resistance in several fungal species is a strong indication that alternative treatments are required. Azoles represent the mainstay of antifungal drugs, and their mode of action involves the binding mode of these molecules to the fungal lanosterol 14a-demethylase target enzyme. In this work, by rational design, we have prepared and characterized four novel organometallic derivatives of the frontline antifungal drug fluconazole (1a4a). All compounds showed excellent in vitro activity against the yeast $C$. robusta, clearly surpassing the progenitor organic drug fluconazole. As anticipated, due to the presence of the ferrocenyl moiety in $\mathbf{1 a - 4 a}$, a modest increase in ROS generation was observed on C. robusta upon treatment. Very importantly, enzyme inhibition and chemogenetic profiling demonstrated that lanosterol $14 \alpha-$ demethylase was the main target of the most active compound of the series, ( $N$-(ferrocenylmethyl)2-(2,4-difluorophenyl)-2-hydroxy-N-methyl-3-(1H-1,2,4-triazol-1-yl)propan-1-aminium chloride, 2a). Transmission electron microscopy (TEM) studies suggested that $\mathbf{2 a}$ induced a loss in wall integrity as well as intracellular features ascribable to late apoptosis or necrosis. The impressive activity of 2a was further confirmed on clinical isolates, where antimycotic potency up to 400 times higher than fluconazole was observed. Also, $2 \mathrm{a}$ showed activity towards azole-resistant strains. This finding is very interesting since the target of $\mathbf{2 a}$ is primarily the same as that of fluconazole, emphasizing the role played by the organometallic moiety. In vivo experiments conducted with $2 \mathbf{2 a}$ at a dose of 10 $\mathrm{mg} / \mathrm{Kg}$ in mice model of Candida infections, while not decreasing fungal burden in the kidney, reduced distal distribution to liver and brain and greatly improved the inflammatory pathology in the kidney and colon, compared to untreated mice.
\end{abstract}




\section{INTRODUCTION}

Mycoses are one of the most common opportunistic infections worldwide, affecting poor as well as industrialized countries. Skin infections (e.g. athlete's foot), for example, affect $20-25 \%$ of the world's population and systemic opportunistic infections are the $4^{\text {th }}$ common cause of bloodstream infections with a lifetime incidence of about $75 \%$ (e.g. in the case of candidiasis). ${ }^{[1]}$ Even if such extensive morbidity does not lead to a high mortality rate, local opportunistic infections together with invasive fungal infections (IFI) have a deep impact on the entire health system because they suppress the immune system and thus, increase the risk to develop other/additional pathologies, which can be fatal to immunosuppresed people. ${ }^{[2]}$ Although potent drugs (e.g. clotrimazole) are applied in antifungal therapy, the infection rate is worryingly increasing every year. Moreover, incidences of resistance to conventional antimycotics are steadily increasing, especially for long term therapies that last from several weeks to months. ${ }^{[3]}$ Three main mechanisms are necessary for antifungal resistance, namely drug target mutations (e.g. mutations of CYP51A1), upregulation of efflux channels (e.g. major facilitator super-family (MFS) and ATP-binding cassette (ABC) channels) and modulation of the stress response (e.g. translational signal pathways, RNA dependent response) ${ }^{[4]}$ Surprisingly, despite a pressing need for novel antifungal agent research and drug development, there is an apparent downturn in such activities, especially in comparison to the vibrant activities in areas such as cancer research.

Cell wall targeting is at the basis of modern antifungal therapy. A selective destabilization or inhibition of cell wall biosynthesis provokes metabolic onset, growth arrest and leads to fungal death. For decades, Amphotericin B deoxycholate $(A m B)$ has been the only available treatment for invasive fungal infections (IFIs). ${ }^{[5]}$ However, during the last ten years, the growing number of IFI incidences has stimulated the introduction of four new classes of antifungal agents, namely polyene-based compounds, azoles, allylamines and echinocandins. ${ }^{[6]}$ Inhibition of cell wall biosynthesis is associated with an accumulation of sterol toxic precursors, cell wall destabilization and cellular stress. During the second half of the $20^{\text {th }}$ century, antifungal research mainly focused on an intensive derivatization of accepted compounds (e.g. from ketoconazole to fluconazole of from AmB to Nystatin and AmB liposomes). ${ }^{[4 \mathrm{~b}-\mathrm{d}]}$ Despite these efforts, to date, only seven compounds are included in the World Health Organization Essential Medicine list (WHO-EM, data of October 2013). ${ }^{[1]}$ Moreover, the benchmark drugs were all discovered almost 20 or more years ago and accounts of ineffective therapies have been reported for several of these agents especially towards Candida albicans and Aspergillus terreus. ${ }^{[1]}$ For this reason and encouraged by the spectacular results obtained with metal complexes (e.g. salvarsan, cisplatin, auranofin, ferroquine, ferrocifen, etc.) in other medicinal fields,\{Biot, 1997 \#818;Jaouen, 2015 \#2259;Barry, 2013 \#2571;Patra, 2017 \#2410;Ong, 2019 \#2607;Gasser, 2012 \#1572;Hartinger, 2012 \#1766;Ott, 2009 \#1892;Patra, 2012 $\# 1624$;Boros, 2020 \#2809\} our groups have decided to assess the potential of such compounds to 
fight fungal infections. ${ }^{[8]}$ These promising examples of metal-based drugs seriously contrasts with the relatively low efforts made in the utilization of such compounds in the field of antifungal therapy. Here, just sporadic reports confirmed the effectiveness of different transition metal complexes (e.g. $\mathrm{Co}(\mathrm{II}), \mathrm{Ni}(\mathrm{II}), \mathrm{Cu}(\mathrm{II}), \mathrm{Pd}(\mathrm{II})$ complexes) bearing a variety of ligands (e.g. azole moieties, thiosemicarbazones, carboxamides, indoles) against several fungal strains or some organometallic moieties bound to active antifungal agents. ${ }^{[9]}$ However, all these studies generally provided only $\mathrm{MIC}_{50}$ values without any further biological investigation. Willing to grasp this opportunity, we embarked on a research program to unveil potent complexes against fungal infections. Herein, we present the identification of a metallocene-based lead compound against such infections. 


\section{RESULTS AND DISCUSSION}

\section{Drug Design}

The two most common antifungal drugs inscribed in the List of Essential Medicine of the WHO and commercially available, namely clotrimazole and fluconazole (Figure 1), belong to the azole class. The molecular target for both those azoles was found to be the enzyme lanosterol-14ademethylase. ${ }^{[9 g]}$ Of utmost interest, mammalian cells use cholesterol instead of ergosterol. This important difference secures high selectivity for the reported antimycotics. ${ }^{[10]}$ Recently, the broad therapeutic applications of clotrimazole as well as fluconazole against skin, genital and invasive fungal infections have generated comparison of primary or cross resistance, especially for Candidiasis. ${ }^{[11]}$ Motivated by the promising results obtained from the ferrocenyl derivatization of organic drugs such as tamoxifen and chloroquine to give ferrocifen and ferroquine, respectively, we envisaged to apply the same concept to fight fungal infections, as previously performed by Brocard, Biot, Jaouen and co-workers. ${ }^{[7 d, 9 i, 12]}$ Fluconazole has been chosen as the parent drug because of its broad spectrum applications (e.g. systemic and topic administration, active against a large number of mycoses). The drug design took into consideration the mode of action of fluconazole and its interaction with the active site of the target enzyme. Computing binding geometry data showed how fluconazole activity is exerted by the interaction of the triazole moiety (i.e. the nitrogen atom) with the haem group present in the active site of the target enzyme (i.e. the iron atom). ${ }^{[13]}$ This interaction is also favored by the presence of the difluorophenyl group that is located in the enzyme active site in (close) proximity to the hydrophobic binding cleft and interacts with it via $\pi-\pi$ stacking (see Figure $\mathrm{S} 1$ in $\mathrm{SI}$ ). ${ }^{[13]}$ On the contrary, the second triazole moiety is involved in non-bonding interactions with several prosthetic groups present in enzyme cavity. Interesting work by Sheng and co-workers demonstrated that organic modification with different functional groups at the C3 atom of fluconazole resulted in an increased activity in relation to the parent drug. ${ }^{[14]}$ Therefore, in the present case, we aimed to derivatize the fluconazole core on the triazole not involved in the binding pocket interaction, replacing it with a ferrocenyl moiety. Ferrocenyl derivatization is proposed to play an important role for the overall biodistribution and uptake (e.g. increasing the lipophilicity of the parent drug) as well as in potentially allowing for an additional redox-induced mode of action as observed for ferrocifen and ferroquine..$^{[\mathrm{d}, 12 \mathrm{a}, 12 \mathrm{~b}]}$ Finally, different alkyl substituents were inserted at the bridging nitrogen in order to garner Structure-Activity Relationship (SAR) information. The target ferrocenyl compounds of this study, namely 1a-4a can be seen in Figure 1. Herein, we name them fluconacenes. 
<smiles>Clc1ccccc1C(c1ccccc1)(c1ccccc1)n1ccnc1</smiles>

Clotrimazole<smiles>OC(Cn1cncn1)(Cn1cncn1)c1ccc(F)cc1F</smiles>

Fluconazole<smiles>[R]N(Cc1ccccc1F)CC(O)(Cn1cncn1)c1ccc(F)cc1F</smiles>

$\begin{array}{ll}\mathrm{R}: \mathrm{H} & =\mathbf{1} \\ \mathrm{R}: \text { Methyl } & =\mathbf{2} \\ \mathrm{R}: \text { Ethyl } & =\mathbf{3} \\ \mathrm{R}: \text { Isopropyl } & =4\end{array}$

Figure 1. Chemical structures of the main antifungal therapeutics present on the market (clotrimazole and fluconazole) and our fluconazenes 1-4.

\section{Synthesis and Characterization}

The synthesis of the new fluconazole ferrocenyl derivatives 1-4 can be visualized in Scheme 1. In the envisioned retrosynthesis, the target compounds would be obtained from the addition of a ferrocenylamine (5) on the epoxide 6 derived from 1,2,4-triazolo-ketone 7 (Scheme 1A). The ferrocenylmethanamines (5a-d) were prepared from ferrocene carboxaldehyde (8) in two steps, following adapted procedures by Tice et al. and Baramee et al. ${ }^{[15]}$ The reaction of the aldehyde (8) with hydroxylamine hydrochloride under basic conditions yielded the oxime (9a). The imines (9b-d) were obtained by stirring 8 in the presence of a solution of the corresponding alkyl amines. The intermediates (9a-d) were reduced to amines (5a-d) with $\mathrm{LiAlH}_{4}$ and $\mathrm{NaBH}_{4}$, respectively. Spectroscopic data of 5a-d matched those reported in the literature (Scheme 1B). ${ }^{[15-16]}$ Epoxide 6 was obtained in $76 \%$ yield through a Corey-Chaykovski epoxidation, by treating ketone $\mathbf{5}$ with a solution of trimethylsulfoxonium iodide ylide (Scheme $1 \mathrm{C}$ ). ${ }^{[13,17]}$ The subsequent epoxide ring opening of $\mathbf{6}$ with the primary and secondary ferrocenemethanamines $(\mathbf{5 a - d})$ yielded compounds $1-$ 4. ${ }^{[16-17]}$ The derivative carrying the methylamine linker (2) was obtained in noticeably higher yield (46\%), than the other derivatives $(1,30 \% ; 3,31 \% ; 4,20 \%)$. This can be attributed to the donating inductive effect of the alkyl groups on the $\mathrm{N}$ atom, which increases the nucleophilic strength of $9 \mathrm{~b}$ d. In contrast, the growing steric hindrance of the ethyl and isopropyl group can explain the decreasing yield within the series of tertiary amines $(2>3>4)$. 
A.
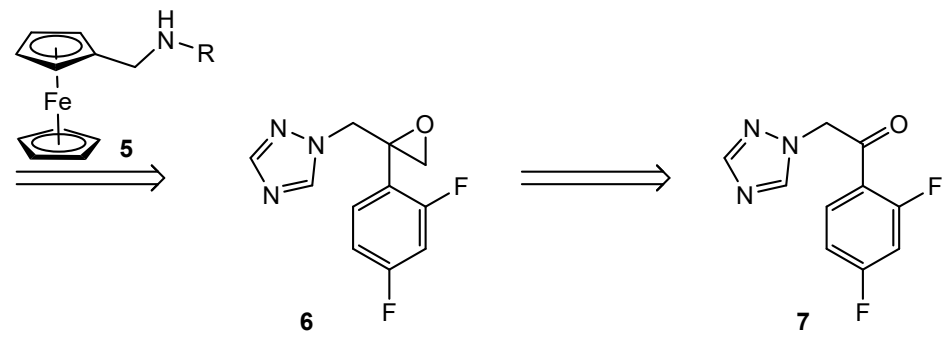

B.

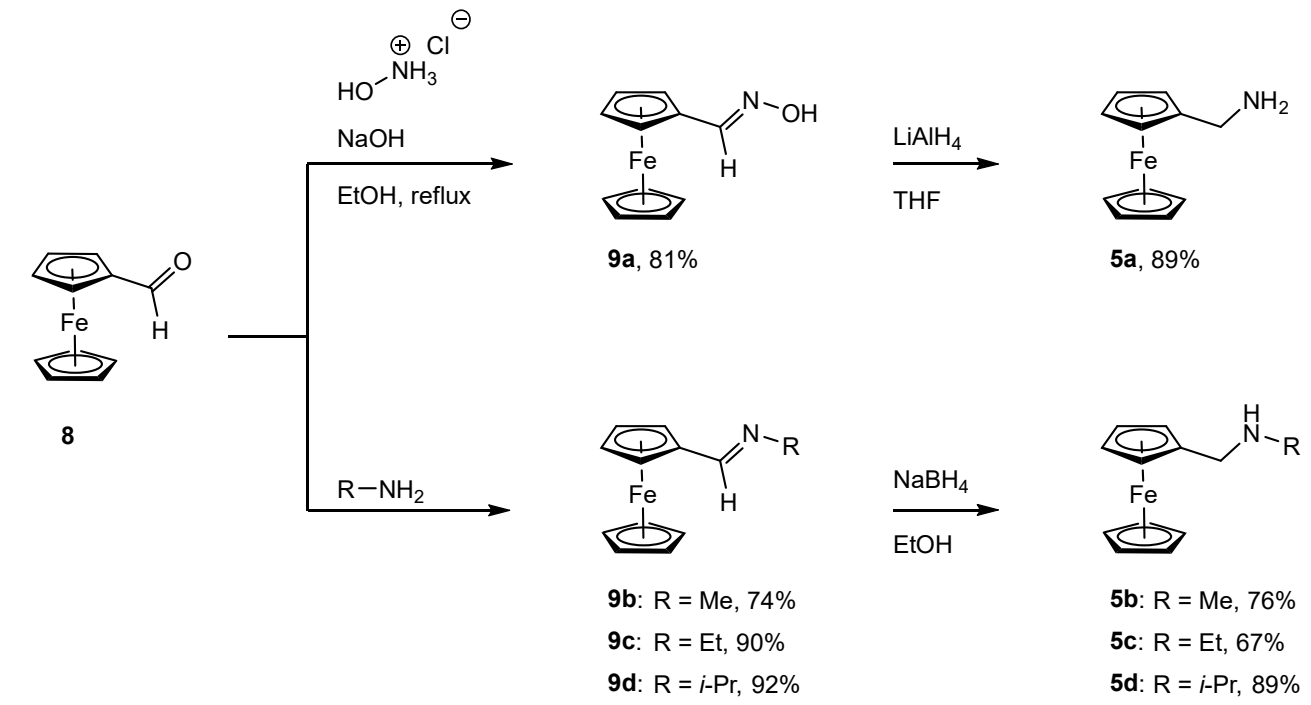

c.<smiles>C[Si](C)(C)O[Na]</smiles>

D.<smiles>[R]CN([R])Cc1ccc(Oc2ccccc2)cc1CC(O)(Cn1cncn1)c1ccc(F)cc1F</smiles>

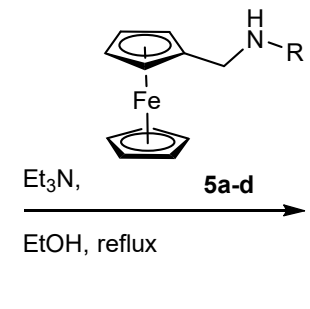<smiles></smiles>

1a: $\mathrm{R}=\mathrm{H}, 82 \%$

2a: $R=M e, 82 \%$

3a: $R=E t, 93 \%$

4a: $\mathrm{R}=i-\mathrm{Pr}, 77 \%$

Scheme 1. A: Retrosynthetic analysis; B: Preparation of the primary (7a) and secondary (7b-d) ferrocenylmethanamines via the oxime $(9 a)$ and imine $(9 b-d)$ intermediates. Overall yields, comprising both steps: 7a, 71\%; 7b, 56\%; 7c, 60\%; 7d, 81\%; C: Synthesis of the organometallic fluconazole derivatives (1-4), which feature an amine-linked ferrocene moiety; D: Synthesis of the hydrochloride salts of 1-4 (1a-4a). 
While fluconazole is achiral with two identical 1,2,4-triazolylmethyl side chains attached to the C2 carbon, our derivatives 1-4 are chiral. Yet, according to molecular docking experiments of chiral fluconazole derivatives by Sheng et al., both $R$ and $S$ isomers, can interact with the active site of the C. albicans CYP51 through a similar binding mode, ${ }^{[18]}$ thus avoiding the need for a chiral separation, or an asymmetric synthesis of each enantiomer. ${ }^{[19]}$ The derivatives 1-4 were converted to the corresponding hydrochloride salts by treatment with $\mathrm{HCl}$ in acetone, following an adapted procedure of Bader et al. (Scheme 1D). ${ }^{[20]}$ Overall, the new complexes 1a-4a were all characterized via ${ }^{1} \mathrm{H},{ }^{13} \mathrm{C}$, ${ }^{19} \mathrm{~F}$ NMR, MS, IR and their purity was confirmed via elemental analysis with a maximal accepted deviation of $0.5 \%$ and UPLC-MS (see Figures S2-S29 in ESI).

\section{Stability}

As potential drug candidates, the hydrochloride salts $1 \mathrm{a}-4 \mathrm{a}$ need to be stable in DMSO, which is the administering medium for in vitro and in vivo studies, as well as in the biological medium: water. It was previously demonstrated that this can be problematic for metal-based drugs.\{Keller, 2020 \#2867\}\{Patra, 2013 \#1972\}\{Hall, 2014 \#2262\} The stability of 1a-4a was assayed by dissolving the salts in deuterated solvents, DMSO- $\mathrm{d}^{6}$ and $\mathrm{D}_{2} \mathrm{O}$, and by monitoring the samples using ${ }^{1} \mathrm{H}$ NMR spectroscopy over a period of 48 hours (see Supplementary Information for details). It is worth mentioning that $\mathbf{1 a}$ and $\mathbf{4 a}$ are only sparingly soluble in $\mathrm{D}_{2} \mathrm{O}$. Generally, all four hydrochloride salts are stable up to 24 hours and only little decomposition was observed for $2 \mathrm{a}$ and $\mathbf{4 a}$ water after $48 \mathrm{~h}$.

\section{In Vitro Biological Screening}

To evaluate the potential of the compounds, we investigated their host toxicity, in vitro mycotoxicity and cellular uptake. The first investigation on the new antimycotic drug candidates involved their possible influence on host cells and their efficiency towards a common fungal model. We investigated the antiproliferative effect of $\mathbf{1 a}-\mathbf{4 a}$ in vitro on human retinal pigment epithelial cell (RPE-1-hTert) and human fibroblast (MRC-5). As expected, fluconazole displayed only moderate effects on cell viability. The complexes displayed all $\mathrm{IC}_{50}$ values in the mid-high micromolar range, following the order of potency: isopropyl > ethyl > methyl > $\mathrm{H}$ (see Table 2). This suggests that a decrease in the size of the $\mathrm{N}$-substituent correlates with a decrease in host toxicity. 
Table 2. Host toxicity of the new fluconacenes against RPE and MRC-5 human cell lines (expressed as $I_{50}$ ) and inhibition of colony formation in $C$. robusta treated with increasing concentrations of the 1a-4a for $24 \mathrm{~h}$ at $30^{\circ} \mathrm{C}$; Fluconazole was used as parent drug for comparative purposes; values are expressed in $\mu \mathrm{M}$.

\begin{tabular}{c|cc|c}
\hline \hline Compound & MRC5 & RPE & C. robusta \\
\hline Fluconazole & $>100$ & $>100$ & $51.0^{ \pm 3.7}$ \\
\hline $\mathbf{1 a}^{\circ}$ & $94.6^{ \pm 2.4}$ & $>100$ & $13.2^{ \pm 3.0}$ \\
\hline $\mathbf{2 \mathbf { a } ^ { \circ }}$ & $32.2^{ \pm 0.1}$ & $74.7^{ \pm 5.2}$ & $3.02^{ \pm 2.18}$ \\
\hline $\mathbf{3 \mathbf { a } ^ { \circ }}$ & $70.4^{ \pm 17.9}$ & $51.2^{ \pm 0.1}$ & $5.86^{ \pm 0.41}$ \\
\hline $\mathbf{4 a}^{\circ}$ & $59.4^{ \pm 2.8}$ & $48.4^{ \pm 2.9}$ & $9.62 \pm 0.56$ \\
\hline \hline
\end{tabular}

To assess the antifungal activity of the new drug candidates, we employed a well-plate based assay recently developed in our group. ${ }^{[8]}$ The first antimycotic investigation was performed on a solid and largely used model, namely wild type C. robusta. Wild type colony of $C$. robusta was cultured and diluted to reach the beginning of the growing phase just before treatment. Agar terrain at increasing concentrations of the target complexes were poured on a 6 or 12-wells plate and the C. robusta culture were then spotted on them and incubated for $24 \mathrm{~h}$ at $30^{\circ} \mathrm{C}$. Interestingly, all compounds displayed an improved antifungal activity compared to the parent drug (e.g. see Figure 2). Small alkyl group-substituted tertiary amines (complexes $\mathbf{2 a}$ and $\mathbf{3 a}$ ) as linkers, showed an improved profile, between 9-17-fold, compared with secondary amines (see Table 2). 


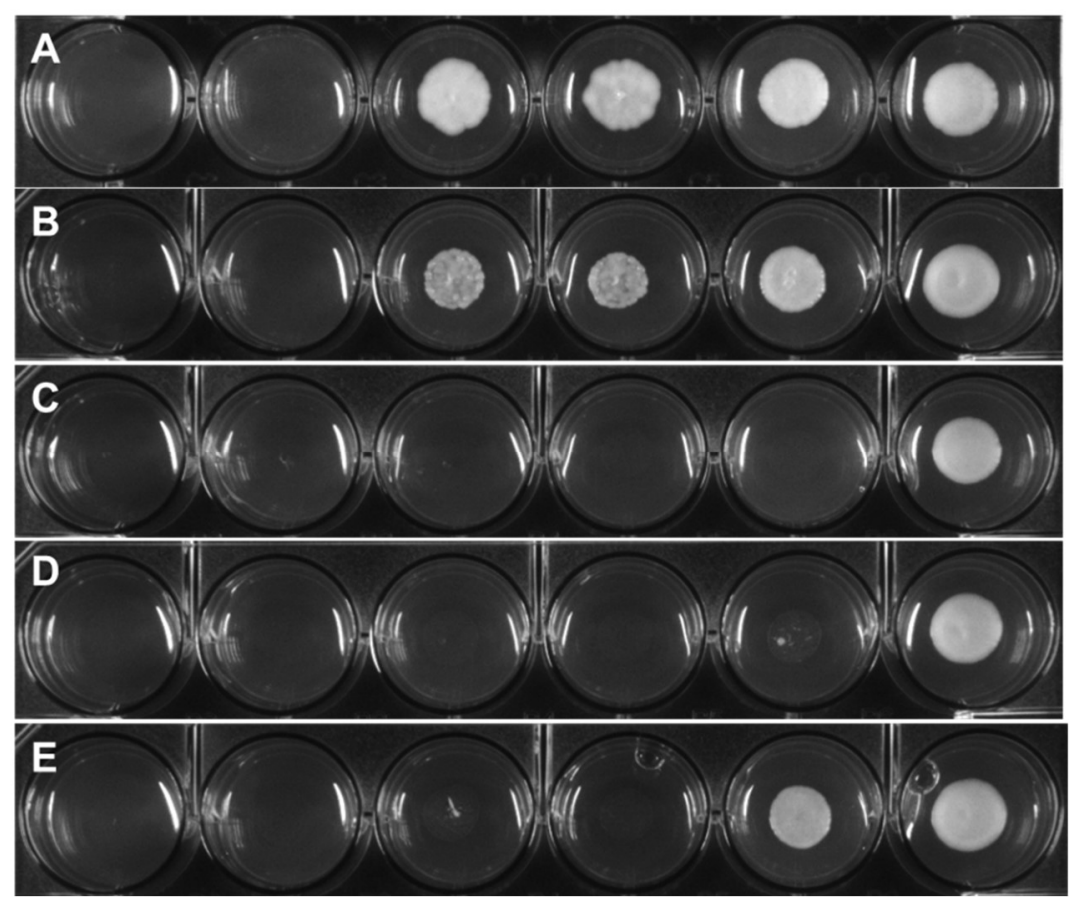

Figure 2. Depicted example of a colony formation inhibition experiment; A) C. robusta treated with fluconazole; B) C. robusta treated with $1 \mathrm{a}$; C) C. Robusta treated with $2 \mathrm{a}$; D) C. robusta treated with 3a; E) C. robusta treated with $\mathbf{4 a .}$

In order to investigate the pharmaco-dynamic profile of the target antimycotic drug candidates, a series of uptake studies in $C$. robusta were performed. The uptake measurements were performed via ICP-MS by detecting the free iron content in the culture medium and normalizing it against the colony density at different incubation times $(1 \mathrm{~h}, 6 \mathrm{~h}$ and $18 \mathrm{~h})$. The results were then compared with the survival rate measured via OD (see Figure 3 ). The uptake of the target complexes followed the order $\mathbf{4 a}<<\mathbf{a}<\mathbf{3} \mathbf{a}<\mathbf{2} \mathbf{a}$ and fits very well with their time-dependent mycotoxicity. This suggests that very hindered groups like an isopropyl or the less lipophilic complex 1a showed a lower efficacy than the one with an alkylic short chain $(2 \mathbf{a}, 3 \mathbf{3})$. 


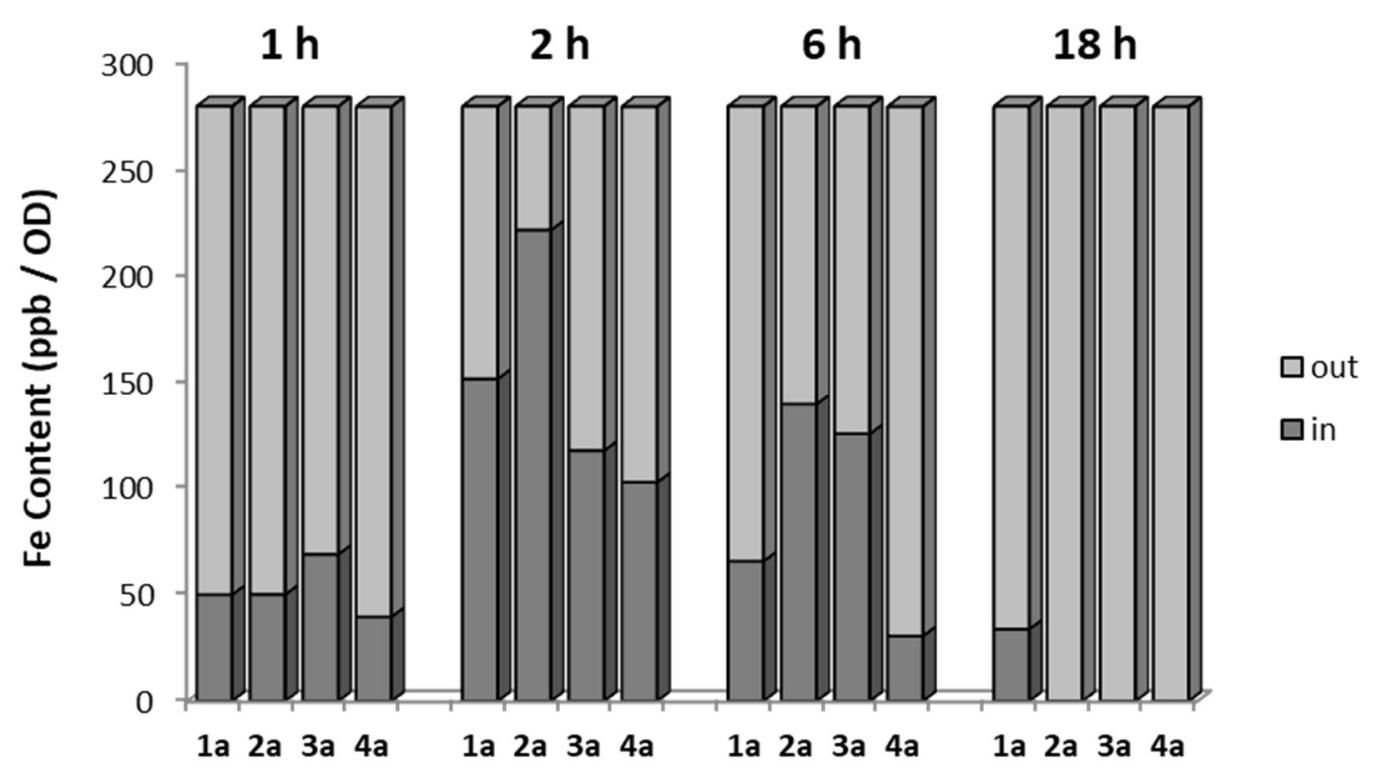

Figure 3. Uptake studies compared to the colony survival rate measured in OD.

In order to assess the possible increase in reactive oxygen species (ROS) level induced by $\mathbf{1 a - 4 a}$, possibly due to the presence of the ferrocenyl moiety, we performed a series of in vitro experiment to measure the ROS level upon treatment on C. robusta. Briefly, C. robusta was cultured in YPD medium overnight before treatment. After that, the fungal concentration was measured and normalized and the cultures were treated with YPD containing $5 \mu \mathrm{M}$ of the different drug candidates at different time frames $(1 \mathrm{~h}, 6 \mathrm{~h}$ and $18 \mathrm{~h})$. After incubation, the colonies were washed, fresh medium containing H2DCF was added and the fluorescence of the hydrolyzed DCF was measured (see Figure 3). While the parent drug fluconazole displayed a weak action at early stages which was increasing with incubation time, the new drug candidates showed a higher ROS level at early stage. However, if from one side the complexes proved to induce an increase of ROS level, the relative ROS induction still remains moderate.

\section{AOX Enzyme Inhibition}

In order to further define the inhibitory capabilities of the compounds, they were also tested against the mitochondrial complex II and III pathway (SQR), two common targets for fungicidal treatment. As can be seen in Figure 4, compound 1a demonstrated no inhibitory activity towards either complex, indicating that the fungicidal activity is indeed due to inhibition of the cytochrome p450 system. However, each of the other compounds demonstrated moderate inhibition, with $\mathrm{IC}_{50}$ values in the micromolar range. 


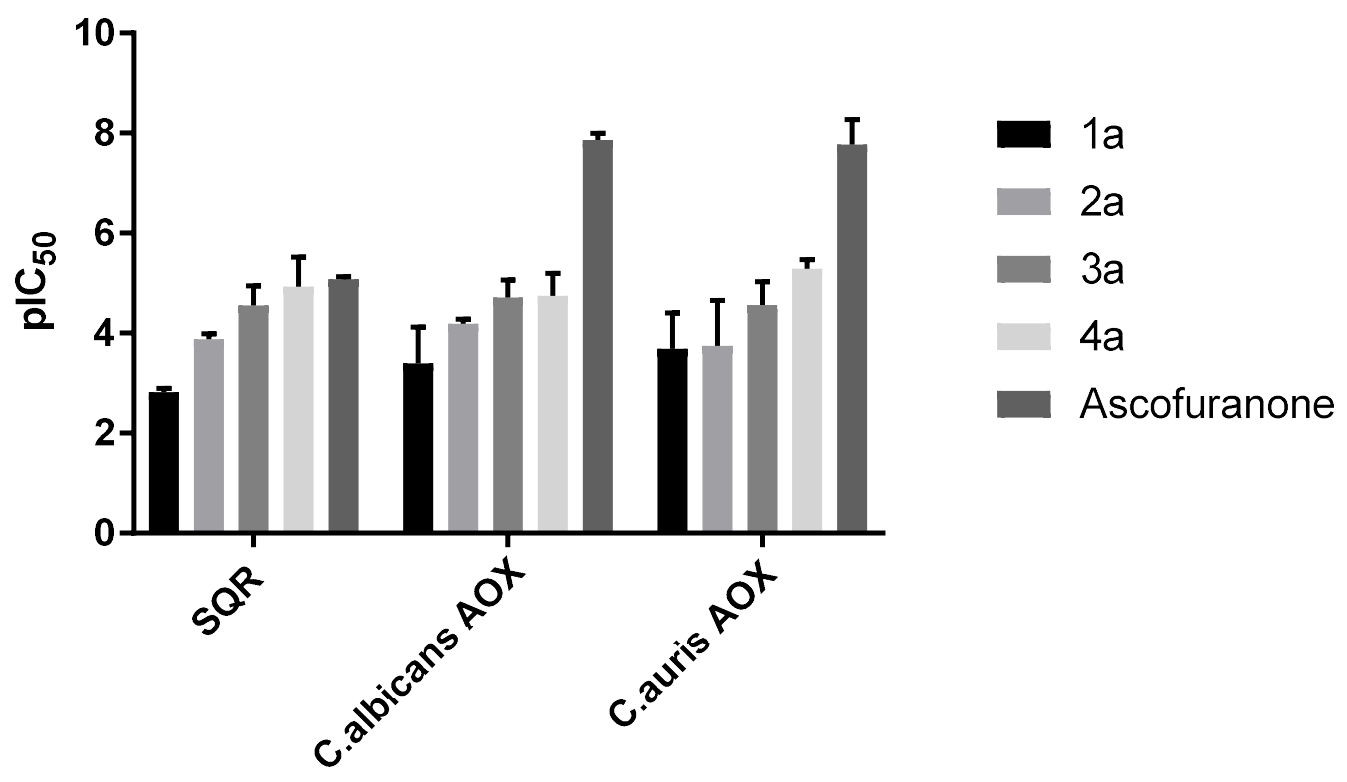

Figure 4. $\mathrm{pIC}_{50}$ values for all compounds against the mitochondrial complex II and III pathway (SQR) and AOX's from Candida albicans and Candida Auris. Ascofuranone is a specific inhibitor of the AOX and used as a control. All data are a mean average of 3 biological replicates \pm SEM.

The compounds were also tested against alternative oxidases (AOX) from both Candida albicans and Candida auris expressed in a recombinant E. coli system. Again, compound 1a demonstrated minimal inhibitory activity towards the AOX's, however compounds $\mathbf{3 a}$ and $\mathbf{4 a}$ showed moderate activity against both AOX's, comparable to values typically seen with non-competitive AOX inhibitors such as salicylhydroxamic acid (SHAM). The parent compound, fluconazole, displayed no inhibition towards either pathway up to a concentration of $100 \mu \mathrm{M}$.

\section{Cytochrome P450 Enzyme Inhibition}

Based on the very promising data obtained with complex $\mathbf{2 a}$, further biological experiments were performed on the best performing complex $2 \mathbf{a}$ to understand further its mechanism of action. Enzyme inhibition studies were performed on different cytochrome P450 enzymes by the service provider Cyprotex $\mathrm{GmbH}$. The new fluconacene $\mathbf{2 a}$ displayed an extremely strong inhibition profile throughout all the series of enzymes, with a larger efficacy in comparison with the parent drug fluconazole, between 4 and 150 fold (see Table 3 ).

Table 3. Enzyme Inhibition studies of different cytochrome P450 enzyme by the compound 2a and its parent drug $\left(\mathrm{IC}_{50}\right.$ in $\left.\mu \mathrm{M}\right)$. Standard deviation shown in upper script.

\begin{tabular}{l|llllll}
\hline \hline Compound & CYP2C9 & CYP2C8 & CYP2C19 & CYP3A4 & CYP1A & CYP2D6 \\
\hline
\end{tabular}




\begin{tabular}{c|cccccc}
\hline Fluconazole & $13.9 \pm 1.63$ & $97.2 \pm 27.9$ & $3.02 \pm 0.34$ & $25.8 \pm 2.45$ & $>100$ & $>100$ \\
$\mathbf{2 a}$ & $3.33 \pm 0.71$ & $0.64 \pm 0.09$ & $<0.4$ & $0.4^{ \pm 0.06}$ & $10.4 \pm 0.41$ & $2.71 \pm 0.12$ \\
\hline \hline
\end{tabular}

\section{Chemogenetic Studies}

In order to investigate the mechanism of action of the new organometallic complex $\mathbf{2 a}$, chemogenomic assays were performed. The strength of the genome-wide chemogenomic screen is reflected in the clear identification of ERG11, encoding lanosterol 14-alpha-demethylase, as the main target of the drug (see Figure 5 and Table S1). ERG11 is a key enzyme of the ergosterol biosynthesis pathway and the primary target of azole antifungals to which the tested compound $\mathbf{2 a}$ belongs. ${ }^{[4 a, 4 c]}$ [4a, 4c] Our assay identified four other heterozygous deletion strains that in previous chemogenomic screens have been shown to be extremely sensitive to azole antifungals; these are SET6, PDR5, MED4 and CDC39. The latter two are essential nuclear genes involved in the regulation of transcription. CDC39 acts as a component of the CCR4-NOT core complex and MED4 encodes a subunit of the RNA polymerase II mediator complex. In Saccharomyces cerevisiae and Candida glabrata the mediator complex is a coactivator of the multiple drug resistance regulator Pdr1 that controls the activation of PDR5 and PDR16. PDR5 is a multidrug $\mathrm{ABC}$ efflux pump that confers resistance to several chemicals including azoles and it mediates transmembrane transport of steorids. PDR16 is a phosphatidylinositol transfer protein involved in sterol biosynthetic process and in resistance to azole drugs. Notably, the phosphatidylinositol signal transduction pathway that controls amongst others cell membrane and cell wall remodelling as well as downstream targets such as DNA repair, was recently suggested to play an important role in phenotypic antifungal drug resistance related to protein synthesis. ${ }^{15}$ Indeed many of the identified deletion strains that are highly sensitive to compound 2a were heterozygous for genes involved in protein synthesis (e.g. ARC1, RPS5, MED4, CDC39, SNF6, CCT5, GSP1) (Fig4, Table S1).

Furthermore, compound 2a targets nuclear genes that are not only essential for RNA transcription, processing and transport (GSP1, MED4, CDC39) but also essential for DNA replication (SLD5), and these together with gene targets functioning in the correct folding of actin and tubulin (CCT5) (Fig 4) point to a possible interaction of compound $2 \mathbf{a}$ with cell division.

Taken together, the genome-wide profiling of the in vivo cellular response to compound $\mathbf{2 a}$ identified candidate protein targets (Fig 4, Table S1), which hint to a mechanism of drug action that goes beyond the main targets of azole antifungals, which might explain its elevated activity towards azole resistant strains (Table 4). 


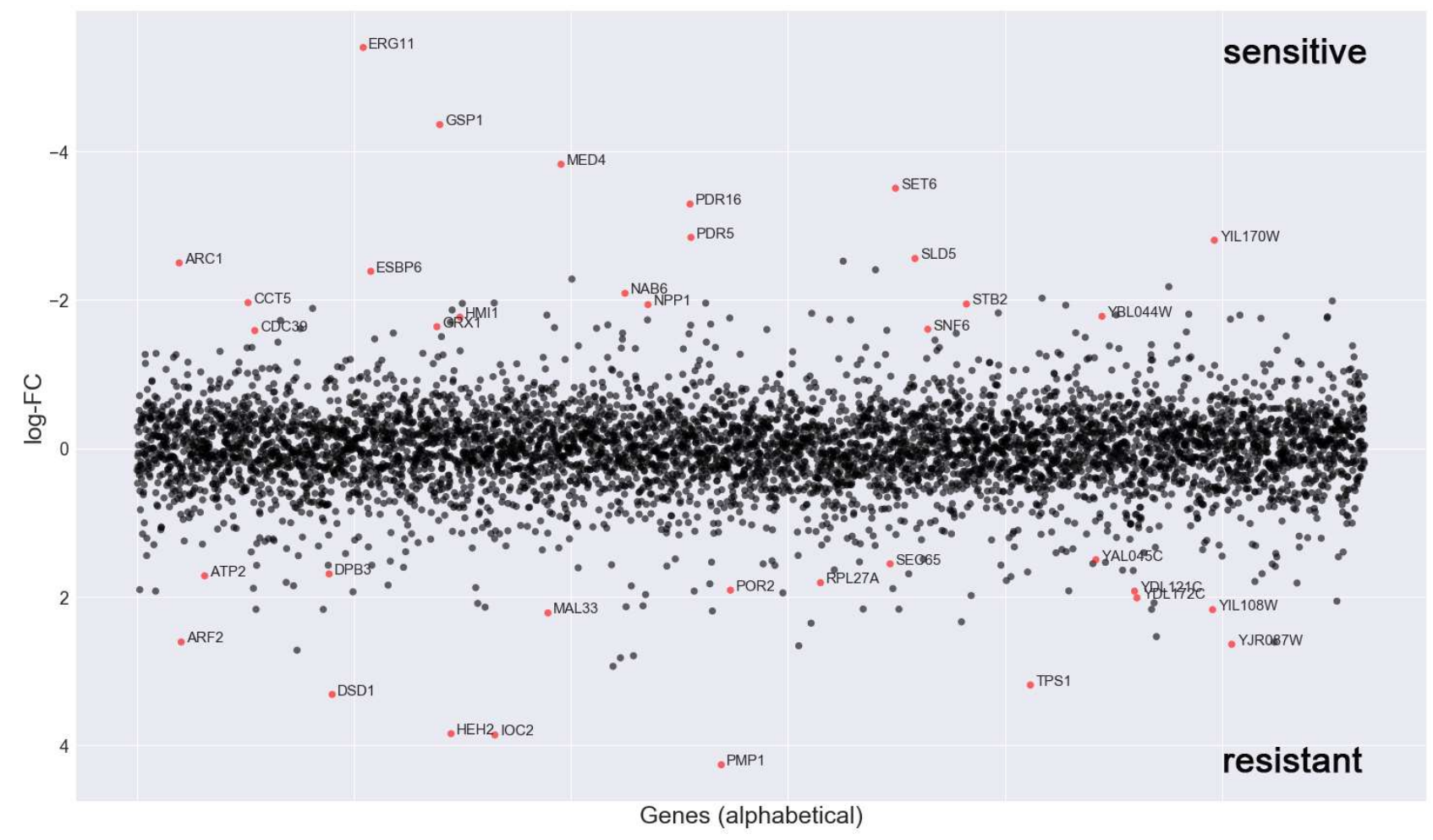

Figure 5. Chemogenomic screen. The pool of tagged 5936 deletion mutants was grown for 20 generations in the presence of compound 2a $(1 \mu \mathrm{M})$ and 1\% DMSO (control). Log2 ratio (control intensity/treatment intensity) was calculated and plotted as a function of gene. The genome-wide readout of heterozygous highly sensitive strains included the known target of azoles, ERG11. (red dots: $\log F C>1.5$ and P.value $>0.01$ ).

\section{Morphology Studies}

The activity of $\mathbf{2} \mathbf{a}$ at the biochemical level (enzyme inhibition studies, chemogenetic profiling) is also mirrored by morphologic features at the ultra-structural level. The morphologic effect on C. robusta upon treatment with the target complex could be conveniently monitored by transmission electron microscopy (TEM). As can be seen in Figure 6, the resulting images (selection of a large pool) suggested that $\mathbf{2 a}$ induces a loss in wall integrity as well as intracellular features ascribable to late apoptosis or necrosis. 

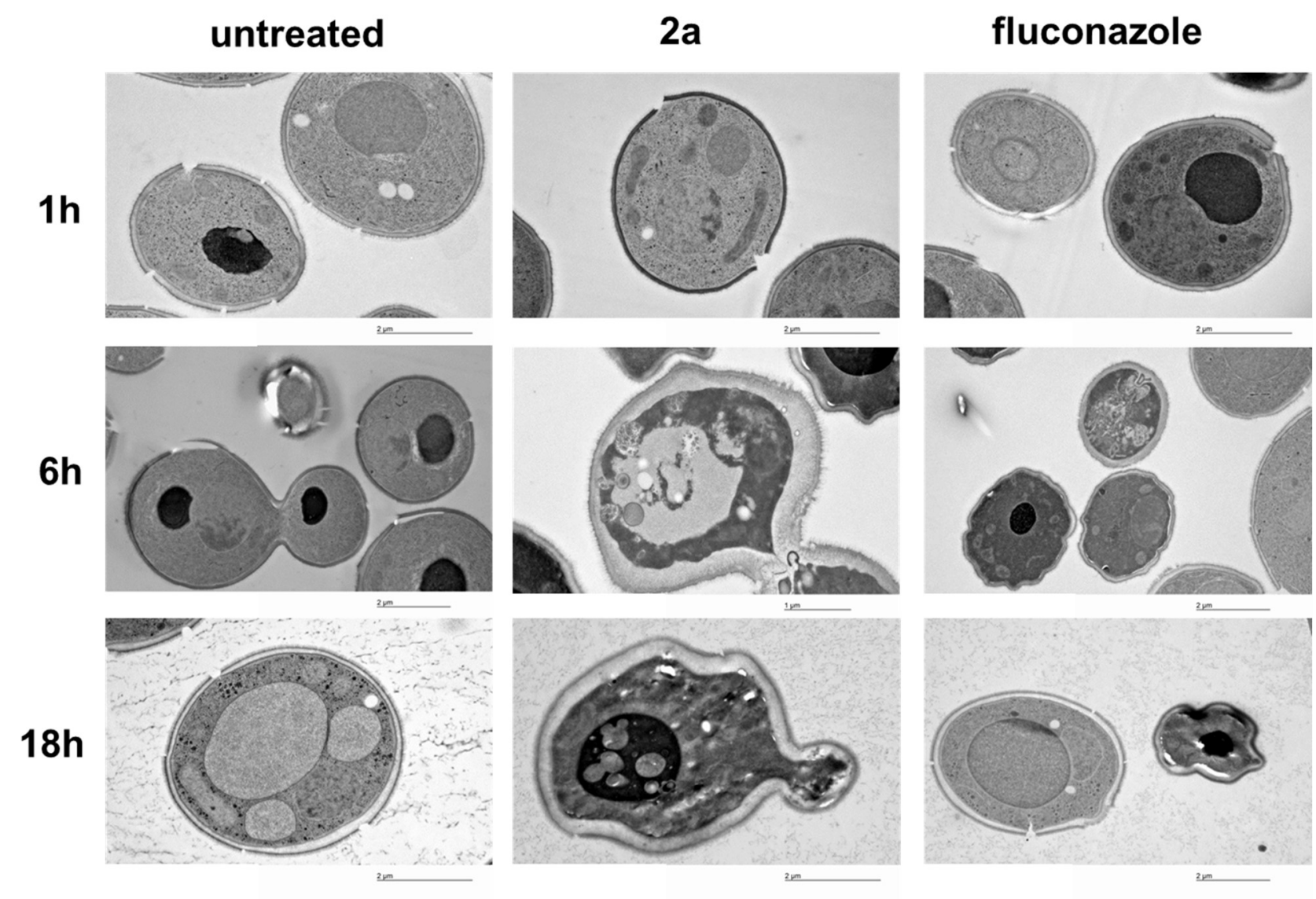

Figure 6. Ultra-structural studies of untreated C. Robusta, treated with $2 \mathrm{a}$ or with Fluconazole tested at $5 \mu \mathrm{M}$ and $30^{\circ} \mathrm{C}$ incubation temperature.

\section{In vitro Antifungal Screening}

Motivated by the promising results obtained for the in vitro antifungal activities, we performed further screening on clinical isolates. We again chose complex 2a since it displayed the best mycotoxicity and, on the other side, showed low cytotoxicity against human cells. We tested this antifungal drug candidate towards Candida albicans and non albicans strains, including fluconazole-resistant (MIC 50 $>100$ ) and other pathogenic fungi, such as Penicillum paneum, Aspergillus glaucus and Trichosporon asahi (see Table 4). Of utmost interest, the new antifungal drug candidate displayed a very strong activity towards almost all investigated strains (only in the case of $A$. glaucus, $C$. parapsilosis, and $C$. albicans MFB005 was the $\mathrm{MIC}_{50}$ not in the nanomolar range). Moreover, the antimycotic potency of the complex did overcome the values of the parent drug up to a factor of 400fold (e.g. P. paneum) and also showed activity towards azole resistant strain (e.g. C. albicans resistant strain). 
Table 4. $\mathrm{MIC}_{50}$ values on a pool of Pathogenic Mycoses treated with 2a

\begin{tabular}{l|cc}
\hline \multicolumn{1}{c|}{ Strains } & MIC $_{50} 2 \mathrm{a}(\mu \mathrm{M})$ & MIC $_{50}$ Fluconazole $(\mu \mathrm{M})$ \\
\hline C. glabrata MFB005 FS4 & $0.07 \pm 0.01$ & $0.4 \pm 0.02$ \\
C. glabrata RTT 199_3 & $1 \pm 0.10$ & $>100$ \\
C. parapsilosis MFB005 FS5 & $0.5 \pm 0.03$ & $0.4 \pm 0.01$ \\
C. parapsilosis MFB070 N1 & $>1$ & $>100$ \\
C. tropicalis RTT35-1 & $0.7 \pm 0.04$ & $>100$ \\
C. tropicalis RTT35-3 & $0.5 \pm 0.03$ & $>100$ \\
C. albicans MFB005 FS3 & $>1$ & $0.4 \pm 0.02$ \\
C. albicans MFB008 MM1 & $0.7 \pm 0.13$ & $>100$ \\
C. albicans YMS 102-2 & $1 \pm 0.15$ & $>100$ \\
C. albicans YMS 102-6 & $0.07 \pm 0.01$ & $0.8 \pm 0.15$ \\
P. paneum MFB042 N1 & $0.25 \pm 0.08$ & $>100$ \\
A. glaucus MFB027 N1 & $>1$ & $0.8 \pm 0.09$ \\
T. asahii MFB034 N1 & $0.13 \pm 0.04$ & $1.6 \pm 0.21$ \\
\hline \hline
\end{tabular}

Motivated by these promising results, we decided to perform in vivo experiments in a mice model of Candida infection. As positive control, fluconazole was used. It significantly improved pathology and cured mice from the infection, as judged by the decreased fungal growth in the kidney and distal dissemination to the brain and liver (see Figure 7A), reduction of the inflammatory pathology in the kidney and colon (see Figure 7B) and downregulation of pro-inflammatory cytokine levels (Fig. 7C). At a dose of $10 \mathrm{mg} / \mathrm{kg}$, while not decreasing fungal burden in the kidney, compound $2 \mathrm{a}$ seemed to diminish distal dissemination to liver and brain (Figure 7A) and, furthermore, greatly improved the inflammatory pathology in the kidney and colon as compared to untreated mice (Figure 7B). Accordingly, the treatment also reduced production of the pro-inflammatory cytokines IL-1 $\beta$, IL-17A, IL-17F, without significantly affecting the levels of anti-inflammatory IL-1Ra and IL-10 (Figure 7C). The anti-inflammatory activity of the compound could not be observed at the lower $(1 \mathrm{mg} / \mathrm{kg})$ dose. This finding suggests that the activity of compound $\mathbf{2 a}$ is clearly dose-dependent, thus offering a plausible explanation as to why a reduction of the fungal burden was not observed. 
A

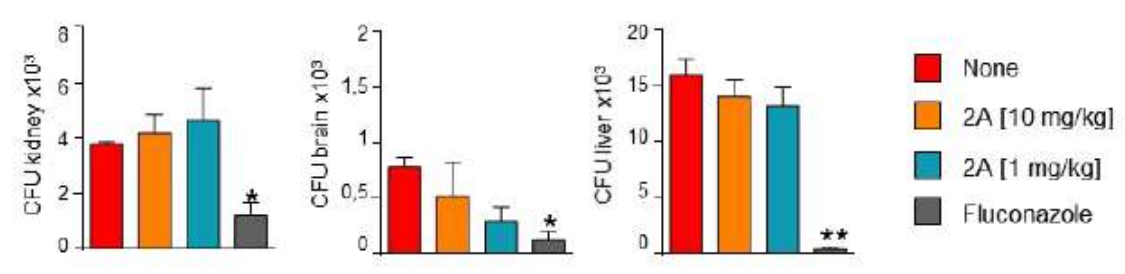

B
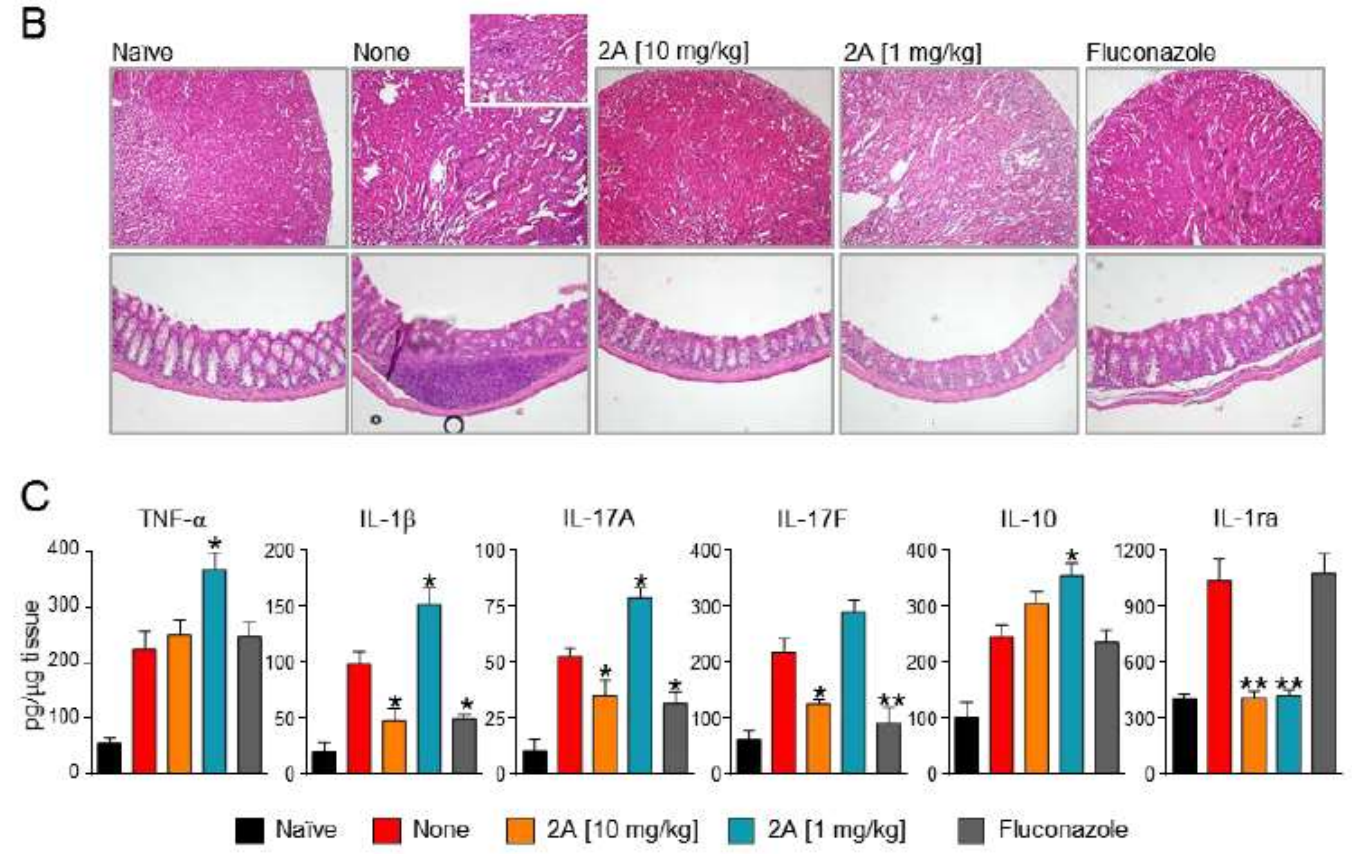

Figure 7. Effect of the compound 2a on Candida albicans systemic infection. C57BL/6 mice were infected via systemic route with $1 \times 10^{6} \mathrm{C}$. albicans yeasts. Compound $2 \mathrm{a}(1$ and $10 \mathrm{mg} / \mathrm{kg}$ ) was administered i.p. daily beginning the day of the infection for 3 consecutive days. Control mice received the diluent alone. (A) Fungal growth. (B) Histological analysis (20X magnification and 40X in the inset). (C) Levels of cytokines in kidney homogenates. Data are expressed as mean $\pm S D$. ${ }^{*} P$ $<0.05$, ${ }^{* \star} \mathrm{P}<0.01$, treated vs untreated C57BL/6 mice. $(\mathrm{n}=8$ mice/group from one experiment). Naïve, uninfected mice. None, untreated mice. 


\section{CONCLUSION}

Four novel ferrocene-based derivatives (1a-4a) of the frontline antifungal drug fluconazole have been synthesized and characterized. All four organometallic derivatives were tested for antifungal activity against $C$. robusta at different concentrations and the half maximal effective concentrations $\left(E C_{50}\right)$ were determined. The derivatives showed an impressively improved activity compared to fluconazole. Importantly, this activity was confirmed on a panel of clinical isolates with the best candidate of this study (complex 2a). An improvement of up to 400 times compared to fluconazole was observed and activity against azole-resistant strains was clearly demonstrated for $\mathbf{2 a}$. During in vivo experiments in mice model of Candida infections $(10 \mathrm{mg} / \mathrm{Kg}$ ), complex 2a was found to diminish distal dissemination to liver and brain and to greatly improve the inflammatory pathology in the kidney and colon, compared to untreated mice.

Overall, this study further demonstrates the potential of organometallic compounds in medicine. By merely inserting a ferrocenyl moiety in a very known drug, namely fluconazole, resistance can be overcome, although the main target of the novel antimycotic agent is the same as that of the organic drug (lanosterol 14a-demethylase), as demonstrated by chemogenetic profiling. The ferrocenyl insertion allows for an additional mode of action. Future work in our groups will be to unveil a more druglike lead molecule. 


\section{ASSOCIATED CONTENT}

\section{Supporting Information}

Experimental procedures, computed binding geometry of fluconazole in the C. albicans CYP51 (Figure S1), Corey-Chaykovski reaction (Scheme S1), possible diastereoisomers/enantiomers of this study (Scheme S2), ${ }^{1} \mathrm{H},{ }^{13} \mathrm{C},{ }^{19} \mathrm{~F}$ NMR, MS, IR spectra and microanalysis (Figures S2-S29), stability of $\mathbf{2 a}$ in DMSO (Figure S30), ROS levels in C. Robusta colonies (Figure S31), top ten sensitive and resistant deletion mutants (Table S1), Complete list of primer sequences (Table S2) are available free of charge via the Internet.

\section{AUTHOR INFORMATION}

\section{Corresponding Authors}

* $\quad$ rubbianiric@gmail.com; $\quad$ tobias.weil@fmach.it; $\quad$ kevin.cariou@chimieparistech.psl.eu; gilles.gasser@chimieparistech.psl.eu

\section{ORCID Numbers}

Tobias Weil: 0000-0003-4448-0782

Noemi Tocci: 0000-0001-6835-5391

Marco Moretto: 0000-0003-4555-7243

Jeannine Hess: 0000-0001-5916-0728

Stefano Ferrari: 0000-0002-6607-215X

Luke Young: 0000-0002-3426-7382

John Spencer: 0000-0001-5231-8836

Tony Moore: 0000-0003-3520-3775

Monica Borghi: 0000-0002-6337-5999

Renga Giorgia: 0000-0002-9762-6493

Luigina Romani: 0000-0002-1356-525X

Gilles Gasser: 0000-0002-4244-5097

\section{Author Contribution}

All authors have given approval to the final version of the manuscript.

\section{Funding Information}

This work was funded by the Swiss National Science Foundation (Grant Sinergia CRSII5_173718, Professorships N PP00P2_133568 and PP00P2_157545 to G.G.), the University of Zurich (G.G), the Stiftung für Wissenschaftliche Forschung of the University of Zurich (G.G.), the Novartis Jubilee Foundation (G.G and R.R.), the Forschungskredit of the University of Zurich (R.R.) and the UBS Promedica Stiftung (G.G and R.R.). This work has received support under the program 
«Investissements d'Avenir » launched by the French Government and implemented by the ANR with the reference ANR-10-IDEX-0001-02 PSL (G.G.). This research was supported by the Autonomous Province of Trento (Accordo di Programma P1611051I (M.M.). Work in ALM's laboratory is supported by BBSRC (BB/L022915/1 and BB/NO10051/1). 


\section{REFERENCES}

[1] a) T. Ohyama, S. Miyakoshi, F. Isono, Antimicrob. Agents Chemother. 2004, 48, 319-322; b) G. Maschmeyer, Int. J. Antimicrob. Agents 2006, 27S, S3-S6; c) A. Mikolajewska, S. Schwartz, M. Ruhnke, Mycoses 2012, 55, 2-16; d) R. Pelletier, J. Peter, C. Antin, C. Gonzalez, L. Wood, T. J. Walsh, J. Clin. Microbiol. 2000, 38, 1563-1568; e) K. Donhuijsen, P. Petersen, W. K. Schmid, Dtsch. Arztebl. Int. 2008, 105, 501-506; fP. N. Lipke, R. Ovalle, J. Bacteriol. 1998, 180, 3735-3740.

[2] a) S. M. Bowman, S. J. Free, Bioessays 2006, 28, 799-808; b) S. Perea, M. J. Ramos, M. Garau, A. Gonzalez, A. R. Noriega, A. del Palacio, J. Clin. Microbiol. 2000, 38, 3226-3230; c) M. P. English, J. Turvey, Brit. Med. J. 1968, 4, 228-230; d) B. Havlickova, V. A. Czaika, M. Friedrich, Mycoses 2008, 51 Suppl 4, 2-15; e) R. P. Hobson, J. Hosp. Infec. 2003, 55, 159-168; fD. A. Enoch, H. A. Ludlam, N. M. Brown, J. Med. Microbiol. 2006, 55, 809-818; g) J. Menzin, J. L. Meyers, M. M. Friedman, J. R. Korn, J. R. Perfect, A. A. Langston, R. P. Danna, G. Papadopoulos, Am. J. Infect. Control 2011, 39, 15-20; h) R. J. Wang, R. F. Miller, L. Huang, Clin. Chest. Med. 2017, 38, 465-477.

[3] H. Hof, Drug. Resist. Update 2008, 11, 25-31.

[4] a) T. Weil, R. Santamaría, W. Lee, J. Rung, N. Tocci, D. Abbey, A. R. Bezerra, L. Carreto, G. R. Moura, M. Bayés, I. G. Gut, A. Csikasz-Nagy, D. Cavalieri, J. Berman, M. A. S. Santos, mSphere 2017, 2, e0016700117; b) L. E. Cowen, W. J. Steinbach, Eukaryotic Cell 2008, 7, 747-764; c) M. A. Pfaller, Am. J. Med. 2012, 125, S3-S13; dC. M. Martel, J. E. Parker, A. G. S. Warrilow, N. J. Rolley, S. L. Kelly, D. E. Kelly, Antimicrob. Agents Chemother. 2010, 54, 4920-4923.

[5] R. S. Shapiro, N. Robbins, L. E. Cowen, Microbiol. Mol. Biol. Rev. 2011, 75, 213-267.

[6] a) D. J. Woods, T. M. Williams, Invert. Neurosci. 2007, 7, 245-250; b) R. Laniado-Laborin, M. N. Cabrales-Vargas, Rev. Iberoam. Micol. 2009, 26, 223-227; c) S. J. Kashyap, V. K. Garg, P. K. Sharma, N. Kumar, R. Dudhe, J. K. Gupta, Med. Chem. Res. 2012, 21, 2123-2132; d) M. A. Ghannoum, L. B. Rice, Clin. Microbiol. Rev. 1999, 12, 501-517.

[7] a) C. Biot, G. Glorian, L. A. Maciejewski, J. Brocard, J. Med. Chem. 1997, 40, 3715-3718; b) G. Jaouen, A. Vessières, S. Top, Chem. Soc. Rev. 2015, 44, 8802-8817, and references therein; c) N. P. E. Barry, P. J. Sadler, Chem. Commun. 2013, 49, 5106-5131; d) M. Patra, G. Gasser Nature Rev. Chem. 2017, 1, 0066, and references therein; e) Y. C. Ong, S. Roy, P. C. Andrews, G. Gasser, Chem. Rev. 2019, 119, 730-796; f) G. Gasser, N. Metzler-Nolte, Curr. Opin. Chem. Biol. 2012, 16, 84-91; g) C. G. Hartinger, N. Metzler-Nolte, P. J. Dyson, Organometallics 2012, 31, 5677-5685; ) hl. Ott, Coord. Chem. Rev. 2009, 253, 1670-1681; i) M. Patra, G. Gasser, N. Metzler-Nolte, Dalton Trans. 2012, 41, 6350-6358.

[8] R. Rubbiani, O. Blacque, G. Gasser Dalton Trans. 2016, 45, 6619-6626.

[9] a) A. Garoufis, S. K. Hadjikakou, N. Hadjiliadis, Coord. Chem. Rev. 2009, 253, 1384-1397; b) N. V. Loginova, T. V. Koval'chuk, N. P. Osipovich, G. I. Polozov, V. L. Sorokin, A. A. Chernyavskaya, O. I. Shadyro, Polyhedron 2008, 27, 985-991; c) T. Mangamamba, M. C. Ganorkar, G. Swarnabala, Int. J. Inorg. Chem. 2014, 2014, 22; d) M. R. Karekal, V. Biradar, M. Bennikallu Hire Mathada, Bioinorg. Chem. Appl. 2013, 2013, 16; e) M. Kaya, E. Demir, H. Bekci, J. Enz. Inhib. Med. Chem. 2013, 28, 885893; f) E. Pahontu, V. Fala, A. Gulea, D. Poirier, V. Tapcov, T. Rosu, Molecules 2013, 18; g) E. RodriguezFernandez, J. L. Manzano, J. J. Benito, R. Hermosa, E. Monte, J. J. Criado, J. Inorg. Biochem. 2005, 99, 1558-1572; h) Z. H. Chohan, H. Pervez, K. M. Khan, C. T. Supuran, J. Enzyme Inhib. Med. Chem. 2005, 20, 81-88; i) C. Biot, N. François, L. Maciejewski, J. Brocard, D. Poulain, Bioorg. Med. Chem. Lett. 2000, 10, 839-841; j) Z. H. Chohan, Appl. Organomet. Chem. 2006, 20, 112-116.

[10] T. Vago, G. Baldi, D. Colombo, M. Barbareschi, G. Norbiato, F. Dallegri, M. Bevilacqua, Antimicrob. Agents Chemother. 1994, 38, 2605.

[11] a) K. Shalini, N. Kumar, S. Drabu, P. K. Sharma, Beilstein J. Org. Chem. 2011, 7, 668-677; b)A. Stütz, Angew. Chem. Int. Ed. 1987, 26, 320-328.

[12] a) G. Jaouen, A. Vessieres, S. Top, Chem. Soc. Rev. 2015, 44, 8802-8817; b) D. Dive, C. Biot, Curr. Top. Med. Chem. 2014, 14, 1684-1692, and references therein; c) S. Sansook, S. Hassell-Hart, C. Ocasio, J. Spencer, J. Organomet. Chem., 2019, 905, 121017. 
[13] X. Chai, J. Zhang, S. Yu, H. Hu, Y. Zou, Q. Zhao, Z. Dan, D. Zhang, Q. Wu, Bioorg. Med. Chem. Lett. 2009, 19, 1811-1814.

[14] S. Krishnan-Natesan, Expert Opin. Pharmacother. 2009, 10, 2723-2733.

[15] a) A. Baramee, A. Coppin, M. Mortuaire, L. Pelinski, S. Tomavo, J. Brocard, Bioorg. Med. Chem. 2006, 14, 1294-1302; b) N. C. Tice, S. Parkin, J. P. Selegue, J. Organomet. Chem. 2007, 692, 791-800.

[16] H. Zhang, F. Xin, W. An, A. Hao, X. Wang, X. Zhao, Z. Liu, L. Sun, Colloids Surf. A 2010, 363, 78-85.

[17] R. S. Upadhayaya, S. Jain, N. Sinha, N. Kishore, R. Chandra, S. K. Arora, Eur. J. Med. Chem. 2004, 39, 579-592.

[18] C. Sheng, W. Zhang, H. Ji, M. Zhang, Y. Song, H. Xu, J. Zhu, Z. Miao, Q. Jiang, J. Yao, Y. Zhou, J. Zhu, J. Lü, J. Med. Chem. 2006, 49, 2512-2525.

[19] M. K. Lindvall, A. M. Koskinen, J. Org. Chem. 1999, 64, 4596-4606.

[20] T. Ader, H.-U. Bischel, B. Gilomen, I. Meyer-Wilmes, M. Sundermeier, 2007. 


\section{Graphical Abstract}

Ferrocene makes it. The derivatization of the known antifungal agent fluconazole with a ferrocenyl moiety allows obtaining a new derivative (2a) with an impressive antimycotic potency up to 400 times higher than fluconazole.<smiles>O=C(O)[C@@H](n1cncn1)C(O)(Cn1cncn1)c1ccc(F)cc1F</smiles>

Fluconazole

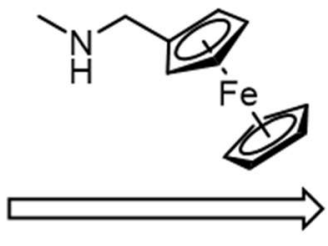

introduce ferrocene

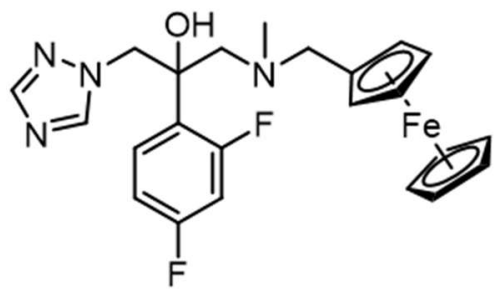

Ferrocenyl analogue
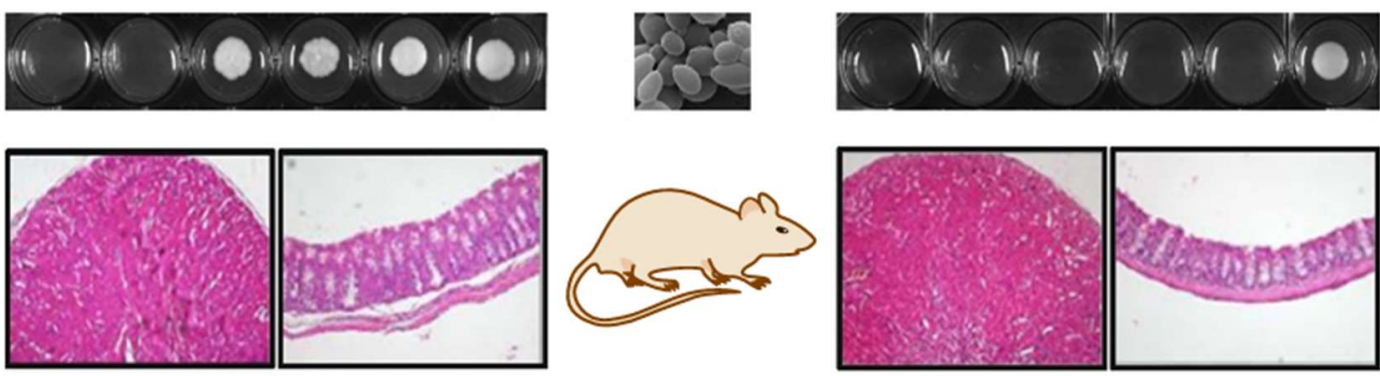